\title{
Microarray Analysis for a Comprehensive Immunological-Status Evaluation during Cancer Vaccine Immune Monitoring
}

\author{
Vladia Monsurrò $^{1,2}$ and Francesco M. Marincola ${ }^{3}$ \\ ${ }^{1}$ Department of Pathology and Diagnostic, University of Verona Medical School, Verona, Italy \\ ${ }^{2}$ Azienda Ospedaliera Universitaria Integrata, Verona, Italy \\ ${ }^{3}$ Infectious Disease and Immunogenetics Section, Department of Transfusion Medicine, Clinical Center, National Institutes of Health, \\ Bethesda, MD 20892, USA \\ Correspondence should be addressed to Vladia Monsurrò, vmonsurro@gmail.com
}

Received 1 July 2011; Accepted 5 August 2011

Academic Editor: Yongqun O. He

Copyright ( $\odot 2011$ V. Monsurrò and F. M. Marincola. This is an open access article distributed under the Creative Commons Attribution License, which permits unrestricted use, distribution, and reproduction in any medium, provided the original work is properly cited.

\begin{abstract}
Anticancer immune responses can be enhanced by immune intervention that promotes complex biological mechanisms involving several cellular populations. The classical immune monitoring for biological-based cancer clinical trials is often based on single-cell analysis. However, the overall effect could be lost by such a reductionist approach explaining the lack of correlation among clinical and immunological endpoints often reported. Microarray technology could give the possibility of studying in a multiparametric setting the immune therapy effects. The application of microarray is leading to an improved understanding of the immune responses to tumor immunotherapy. In fact, analysis of cancer vaccine-induced host responses using microarrays is proposed as valuable alternative to the standard cell-based methods. This paper shows successful examples of how high-throughput gene expression profiling contributed to the understanding of anticancer immune responses during biological therapy, introducing as well the integrative platforms that allow the network analysis in molecular biology studies.
\end{abstract}

\section{Microarray Analysis and Immune System Functional Status in Antitumor Biological Therapies}

Immune-based therapies that enhance the natural antitumor immune response represent an attractive therapeutic approach. However, the ongoing development of such therapies, which includes vaccines, use of immune modulators, DC therapy, and adoptive immunotherapy, has been labored by the lack of a general method for the assessment of quantity, functional activity, and overall effect of the immune response. Immunologic monitoring of cancer immunotherapy trials has been historically focused on cell-based assays to quantify tumor antigen-specific cellular immune responses via phenotypic characterization or functional analysis associated to an antigen-specific response [1-3]. A boost to the immune monitoring derived from the development of peptide-MHC Class I tetramers has become a key tool for the antigen-specific cancer vaccine immune monitoring to ex vivo identify, count, and isolate antigen-specific $\mathrm{T}$ cells directly from blood patient samples $[4,5]$. However, the major disadvantages of such assay compared to other assays such as ELISPOT or intracellular cytokine flow cytometry are the low number of different specificities that can be assessed at the same time and the lack of functional characterization. This could lead to paradoxical coexistence of a tumor-specific immune response and the progression of the disease $[6,7]$. Even more, since immune therapy impacts multiple facets of the immune response, those broad complex interactions could easily be lost if the monitoring is focused and limited to the antigen-specific phenotypic immune response analysis [8]. This was the case of tetramer-specific vaccine-induced cells in a peptide-based cancer immunotherapy setting, since the phenotypic characterization was showing a paradoxical presence of tetramer-specific cells $[2,6]$, able to produce interferon gamma [2] associated to clinical failure of the therapy [8]. The interplay between cancer and immune system, in the setting of cancer vaccine immune monitoring, 
must consider that both cancer and all the portion of the immune system may affect each other's functionality by direct contact or through the production of molecules with immune modulatory properties. Such complexity could only be recently appreciated in all its extent by high-throughput tools capable of providing a global view of biological processes [9]. Thanks to microarray analysis, we were able to understand the paradoxical coexistence of tumor antigenspecific cells and tumor in the same host by describing the quiescent status of the antigen-specific T cells $[6,7]$. In order to dissect this complex interaction in a single-cell functional focus, recently, the group of Heath proposed the use of clinical microchip for the evaluation of single immune cell functionality [10]. This innovative single-cell-based analysis can lead to the resolution of the contribution of each single cell to the overall effect in response to therapy, suggesting the possible use as a potential application to the field of immune monitoring for cancer vaccines as well [10]. This study however can be considered complementary to the information that is generated through microarray. In fact, the array technology leads to complementary information since it is able to enlarge the view on the final overall occurring biological phenomena during the vaccination protocol as resultant of the action of each single-cell activity.

\section{Use of Microarray to Reveal Biological Response to Cancer Therapies}

In human, one of the most characterized immune response to a biological therapy through microarray technology is the response to interferon alpha (IFN alpha), one of the most used cytokine in clinics with the goal of activating the immune system in adjuvant setting. Zimmerer et al. first described the transcriptional response of $\mathrm{T}$ cells, natural killer cells, and monocytes to IFN-alpha and characterized the transcriptional profiles of PBMCs from melanoma patients undergoing high dose of IFN-alpha immunotherapy. In his study, he analyzed the profile of in vitro and in vivo immune cells stimulated with interferon alpha showing a similar activation profile in vivo and in vitro. He proposed a signature associated to the effect of this therapy on the different immune cell subpopulations, suggesting the microarray analysis of in vitro stimulated PBMCs as useful predictor of response tool [11]. Recently, gene profiling analysis in similar setting was performed by Berardelli's group on PBMC isolated from melanoma patients and healthy individuals 24 hours after each repeated injection for a low-dose IFN-alpha, administered as vaccine adjuvant in two separate clinical trials. This study was able to characterize IFN-alpha mechanisms of action, finding a core signature of these cytokines in the peripheral blood of IFN-alpha-treated individuals, as well as on the existence of blood genomic and proteomic markers of low-dose IFNalpha administered as a vaccine adjuvant. Those results provide new insights into the identification of a welldefined molecular signature as biomarker of low-dose IFNalpha administered as immune adjuvant [12]. A successful application of immune monitoring through microarray technology is for example the study of tumor response to an attenuated recombinant vaccinia virus (GLV-1h68) that selectively colonizes established human xenografts inducing their complete regression. In this model, tumor rejection was associated in vivo with activation of interferon-stimulated genes and innate immune host-effector functions [13]. In this study, through the use of array technology, was clearly demonstrated the synergy between the innate and adaptive immune response required for successful tumor rejection. In recent papers, the microarray technology was used in order to monitor the immune response to cytokine-based immunotherapy.

In a different setting of experimental model, using a mouse neuroblastoma cell line, Kohler demonstrated that vaccination of tumor-free mice with a cell-based vaccine leads to productive immunity and resistance to tumor challenge, while vaccination of tumor-bearing mice does not. The T-cell immunity induced by this vaccine, as measured by in vitro assays, is amplified by the depletion of Treg. Our goal is to understand this barrier to the development of protective cellular immunity. mRNA microarray analyses of $\mathrm{CD} 8(+) \mathrm{T}$ cells from naïve or tumor-bearing mice undergoing vaccination were carried out with or without administering anti-CD25 antibody. Gene-expression pathway analysis revealed the presence of CD8(+) T cells expressing stem cell-associated genes early after induction of productive antitumor immunity in tumor-free mice, prior to any phenotypic changes, but not in tumor-bearing mice. These data demonstrate that early after the induction of productive immune response, cells within the CD8(+) T-cell compartment adopt a stem cell-related genetic phenotype that correlates with increased antitumor function $[14,15]$. The microarray analysis has been able to clarify as well the immunological functional status of cancer patient's immune system. Lee et al. were able to elucidate the nature of the molecular bases of the immune dysfunction on melanoma patient by comparing PBMCs from 12 healthy donors to PBMCs from 12 melanoma patients. A high percentage of significantly altered genes in $\mathrm{T}$ cells and $\mathrm{B}$ cells from melanoma patients belongs to interferon- (IFN-) stimulated genes family, and the data was functionally validated since the median percentage of lymphocytes that phosphorlate STAT1 in response to IFN-alpha was significantly reduced in melanoma patients compared to healthy controls in Phosflow analysis. Interestingly, this immune dysfunction was reverted by administering high dose of interferon. In this study, the application of microarray technology has led to the definition of a new defects in interferon signaling as a predominant mechanisms of immune dysfunction in cancer [16]. The vision of the use of microarray as standard immune monitoring tool was explored in the study by Dendron where normal-donor immune responses to CMV, influenza, tetanus and C. albicans antigens were investigated in order to identify a set of genes that could become a model for monitoring of antitumor immune response induced by antigen-specific in vivo immune therapy. In this study, healthy donors PBMCs were stimulated for 24 hours with the respective antigen and analyzed for changes in gene expression. Of all the genes differentially expressed, 85 were common to 
the four different stimuli with CD40, showing the strongest and most consistent upregulation upon antigen stimulation of all genes analyzed. These results demonstrate the utility of microarrays to investigate antigen-induced changes in gene expression in PBMCs and provide support for the use of microarrays in the immune monitoring of subjects participating in therapeutic cancer vaccine trials [17].

\section{New Software for Network Analysis for the Translation from Dataset to Biological Meaning}

High-throughput technologies generate a big amount of data leading to a parallel need of implement computational platforms that integrate pathway, protein-protein interaction, genome, and literature mining data to result in comprehensive networks for a list of genes or proteins. In a recent and comprehensive review, Thomas and Bonchev from the center for the study of biological complexity, Virginia Commonwealth University, summarized the most used software for network analysis in living cells, the nodes in which are represented as protein-protein interactions or gene regulatory networks [18]. They compared the three major software packages for network analysis used CYTOSCAPE, INGENUITY, and PATHWAY STUDIO, and in the same paper, they compared in detail their characteristics as well as their associated databases. For example, characteristic and unique for the ingenuity pathway analysis is the database for human genes/peptides created from manually evaluated literature searches (Ingenuity ExpertAssist Findings). Not included in the previous paper but of high interest is a tool made available from the group of computational Systems Biology Laboratory, Institute of Biomedicine and Genome-Scale Biology Program at University of Helsinki. Dr. Hautaniemi and Dr. Laakso have implemented a computational platform (Moksiskaan) that is now able to generate hypothetical pathways for a set of genes or proteins as well as to estimate their activation statuses using regulation information in pathway repositories. The result of this type of analysis is a document that provides description of the query genes and biological processes. An important function is the generation of hypothetical drug targets that can sensibly empower a translational type of research in the oncologic field. Moksiskaan networks results can ultimately be analyzed as well in Cytoscape for further personalized analysis. (Moksiskaan and user guide are freely available under GNU General Public License at http://csbi.ltdk.helsinki.fi/moksiskaan/ [19].)

\section{Acknowledgments}

The authors are thankful to Dr. Silvia Coin and Miss Svjetlana Raus for help in proofreading the paper. V. Monsurro was financed by grants from AIRC and Italian Ministry of Health and SC is recipient of an Italian Ministry of Health fellowship.

\section{References}

[1] P. P. Peethambaram, M. E. Melisko, K. J. Rinn et al., "A phase I trial of immunotherapy with lapuleucel-T (APC8024) in patients with refractory metastatic tumors that express HER2/neu," Clinical Cancer Research, vol. 15, no. 18, pp. 59375944, 2009.

[2] V. Monsurrò, D. Nagorsen, E. Wang et al., "Functional heterogeneity of vaccine-induced CD8+ T cells," Journal of Immunology, vol. 168, no. 11, pp. 5933-5942, 2002.

[3] M. B. Nielsen, V. Monsurro, S. A. Migueles et al., "Status of activation of circulating vaccine-elicited CD8+ T cells," Journal of Immunology, vol. 165, no. 4, pp. 2287-2296, 2000.

[4] S. Seiter, V. Monsurro, M. B. Nielsen et al., "Frequency of MART-1/MelanA and gp100/PMel17-specific T cells in tumor metastases and cultured tumor-infiltrating lymphocytes," Journal of Immunotherapy, vol. 25, no. 3, pp. 252-263, 2002.

[5] V. Monsurrò, M. B. Nielsen, A. Perez-Diez et al., "Kinetics of TCR use in response to repeated epitope-specific immunization," Journal of Immunology, vol. 166, no. 9, pp. 5817-5825, 2001.

[6] V. Monsurrò, E. Wang, Y. Yamano et al., "Quiescent phenotype of tumor-specific CD8+ T cells following immunization," Blood, vol. 104, no. 7, pp. 1970-1978, 2004.

[7] V. Monsurrò, E. Wang, M. C. Panelli et al., "Active-specific immunization against melanoma: is the problem at the receiving end?" Seminars in Cancer Biology, vol. 13, no. 6, pp. 473-480, 2003.

[8] S. A. Rosenberg, J. C. Yang, and N. P. Restifo, "Cancer immunotherapy: moving beyond current vaccines," Nature Medicine, vol. 10, no. 9, pp. 909-915, 2004.

[9] V. Monsurrò and F. M. Marincola, "Gene profiling for the prediction of tumor response to treatment: the case of immunotherapy," Advances in Experimental Medicine and Biology, vol. 593, pp. 86-94, 2007.

[10] C. Ma, R. Fan, H. Ahmad et al., "A clinical microchip for evaluation of single immune cells reveals high functional heterogeneity in phenotypically similar T cells," Nature Medicine, vol. 17, no. 6, pp. 738-743, 2011.

[11] J. M. Zimmerer, G. B. Lesinski, A. S. Ruppert et al., "Gene expression profiling reveals similarities between the in vitro and in vivo responses of immune effector cells to IFN- $\alpha$," Clinical Cancer Research, vol. 14, no. 18, pp. 5900-5906, 2008.

[12] E. Aricò, L. Castiello, F. Urbani et al., "Concomitant detection of IFN $\alpha$ signature and activated monocyte/dendritic cell precursors in the peripheral blood of IFN $\alpha$-treated subjects at early times after repeated local cytokine treatments," Journal of Translational Medicine, vol. 9 article 67, 2011.

[13] A. Worschech, N. Chen, Y. A. Yu et al., "Systemic treatment of xenografts with vaccinia virus GLV-1h68 reveals the immunologic facet of oncolytic therapy," BMC Genomics, vol. 10, article 301, 2009.

[14] M. E. Kohler, W. H. D. Hallett, Q. R. Chen, J. Khan, B. D. Johnson, and R. J. Orentas, "Early expression of stem cellassociated genes within the CD8 compartment after treatment with a tumor vaccine," Cellular Immunology, vol. 265, no. 1, pp. 65-73, 2010.

[15] E. Wang, "Microarrays Gene expression profiling approaches for the monitoring of anti-cancer immune responses," in Analyzing T Cell Responses, D. Nagorsen and F. M. Marincola, Eds., pp. 285-301, Springer, Amsterdam, The Netherlands, 2005.

[16] R. J. Critchley-Thorne, N. Yan, S. Nacu, J. Weber, S. P. Holmes, and P. P. Lee, "Down-regulation of the interferon signaling 
pathway in $\mathrm{T}$ lymphocytes from patients with metastatic melanoma," PLoS Medicine, vol. 4, no. 5, pp. 0897-0911, 2007.

[17] K. W. Makar, P. Aaron, and L. A. Jones, "Application of microarray technology to the immune monitoring of therapeutic cancer vaccines," in Proceedings of the AACR Annual Meeting, Anaheim, Calif, USA, 2005.

[18] S. Thomas and D. Bonchev, "A survey of current software for network analysis in molecular biology," Human Genomics, vol. 4, no. 5, pp. 353-360, 2010.

[19] M. Laakso and S. Hautaniemi, "Integrative platform to translate gene sets to networks," Bioinformatics, vol. 26, no. 14, Article ID btq277, pp. 1802-1803, 2010. 


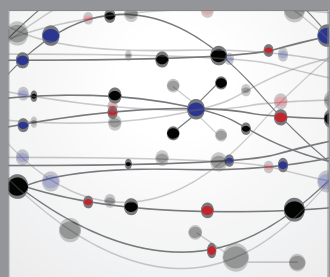

The Scientific World Journal
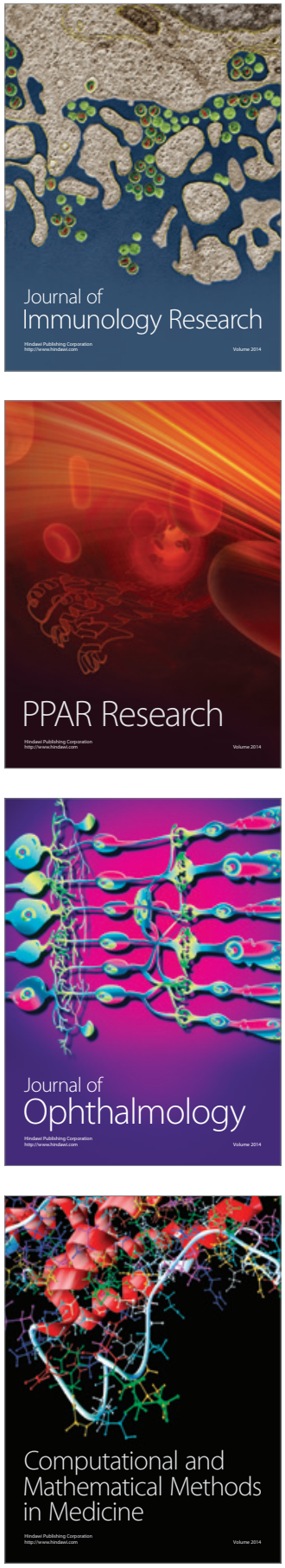

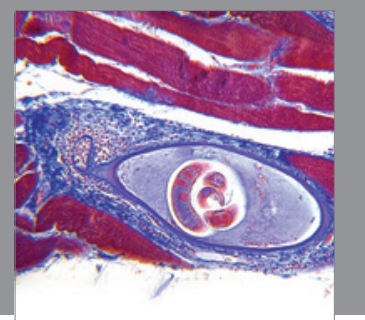

Gastroenterology

Research and Practice
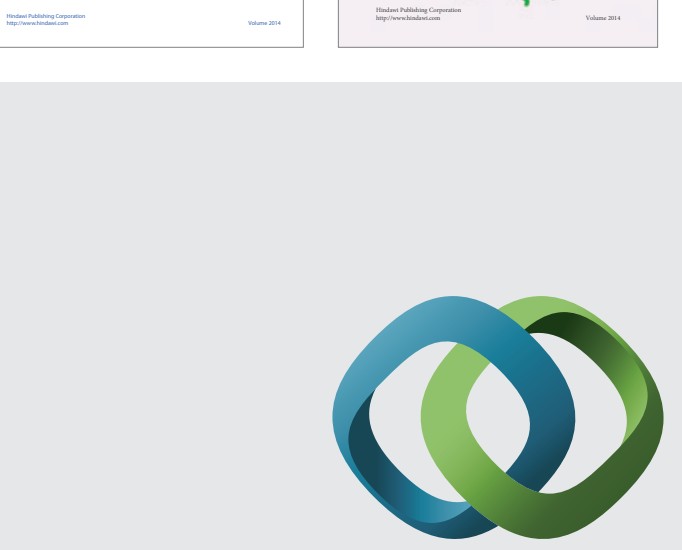

\section{Hindawi}

Submit your manuscripts at

http://www.hindawi.com
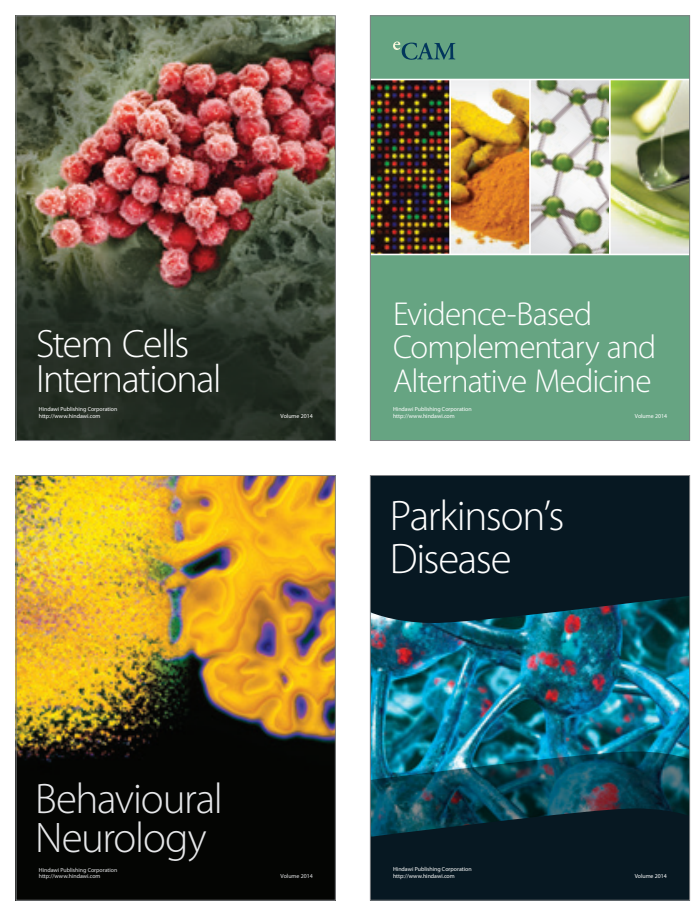

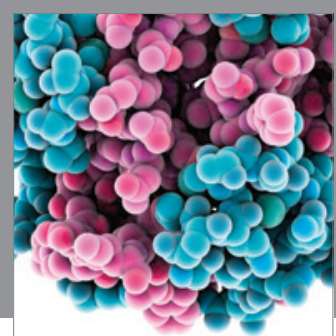

Journal of
Diabetes Research

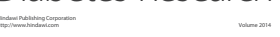

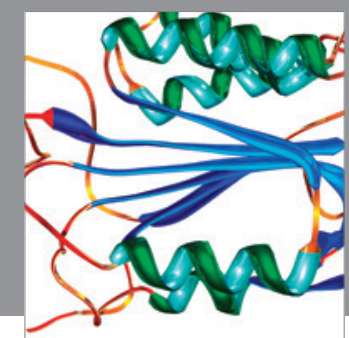

Disease Markers
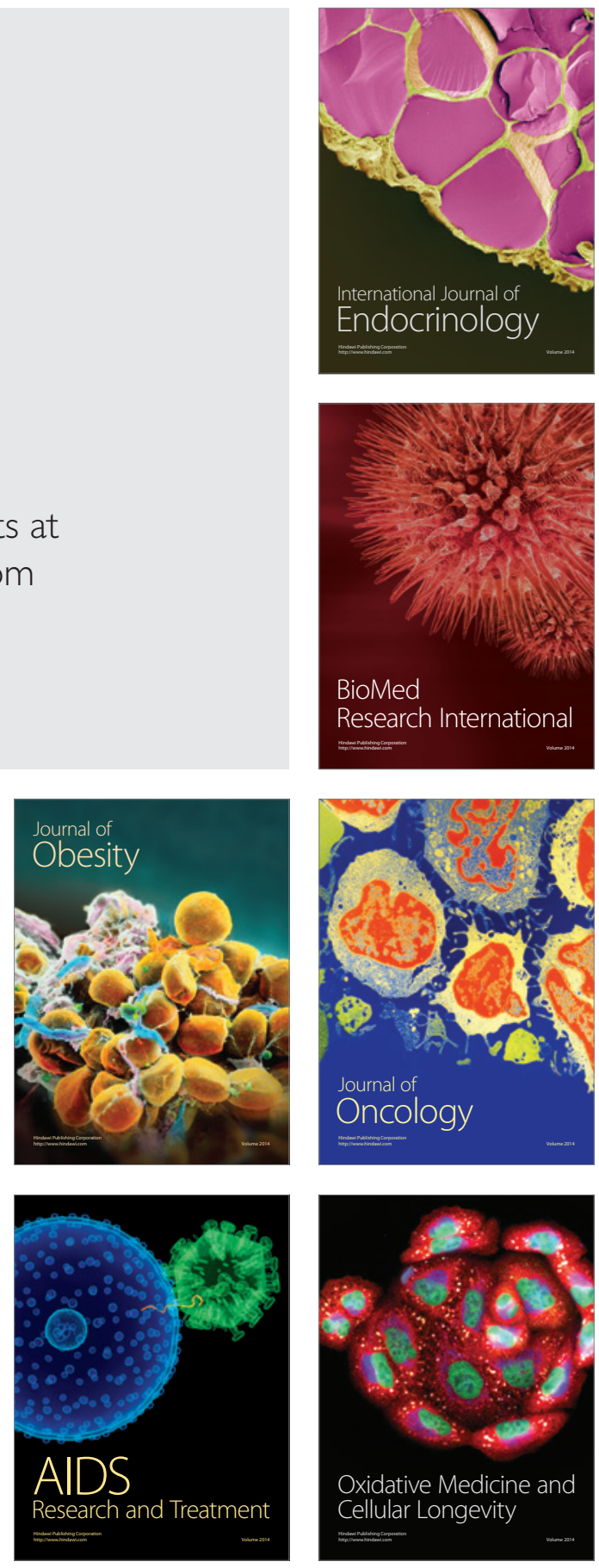